\title{
OPEN Optical vagus nerve modulation of heart and respiration via heart-injected retrograde AAV
}

\author{
Arjun K. Fontaine ${ }^{1,3,8 凶}$, Gregory L. Futiaa, ${ }^{1,8}$, Pradeep S. Rajendran ${ }^{4,5}$, Samuel F. Littich ${ }^{1,3}$, \\ Naoko Mizoguchi $i^{2,7}$, Kalyanam Shivkumar ${ }^{4,5}$, Jeffrey L. Ardell ${ }^{4,5}$, Diego Restrepo ${ }^{2,9}$, \\ John H. Caldwell ${ }^{2,9}$, Emily A. Gibson ${ }^{1,9}$ \& Richard F. ff Weir ${ }^{1,3,6,9}$
}

Vagus nerve stimulation has shown many benefits for disease therapies but current approaches involve imprecise electrical stimulation that gives rise to off-target effects, while the functionally relevant pathways remain poorly understood. One method to overcome these limitations is the use of optogenetic techniques, which facilitate targeted neural communication with light-sensitive actuators (opsins) and can be targeted to organs of interest based on the location of viral delivery. Here, we tested whether retrograde adeno-associated virus (rAAV2-retro) injected in the heart can be used to selectively express opsins in vagus nerve fibers controlling cardiac function. Furthermore, we investigated whether perturbations in cardiac function could be achieved with photostimulation at the cervical vagus nerve. Viral injection in the heart resulted in robust, primarily afferent, opsin reporter expression in the vagus nerve, nodose ganglion, and brainstem. Photostimulation using both onephoton stimulation and two-photon holography with a GRIN-lens incorporated nerve cuff, was tested on the pilot-cohort of injected mice. Changes in heart rate, surface electrocardiogram, and respiratory responses were observed in response to both one- and two-photon photostimulation. The results demonstrate feasibility of retrograde labeling for organ targeted optical neuromodulation.

Improved techniques are necessary for selective modulation of peripheral nerve fibers, particularly within the autonomic nervous system. Effort in recent years has increasingly been aimed at targeting neural pathways of abdominal and thoracic organs for therapeutic neuromodulation ${ }^{1-3}$. While pharmacologic treatments have poorly confined spatial and temporal precision, the premise underlying initiatives in 'bioelectronic medicine' or 'electroceuticals' is compelling; tapping into the neural circuitry driving organ function may enable approaches for disease treatment pathways unachievable or far less precisely achievable with pharmacologic approaches.

Vagus nerve stimulation (VNS) interventions using electrode-based techniques have shown clinical efficacy in treating numerous diseases. VNS is clinically-approved for the treatment of epileptic seizures ${ }^{4,5}$ and depression ${ }^{6}$, and clinical studies demonstrate benefit in treating inflammatory disorders such as Crohn's disease ${ }^{7}$ and rheumatoid arthritis ${ }^{8}$, as well as hypertension ${ }^{9}$ and obesity ${ }^{10}$. For the heart, clinical studies point to therapeutic potential in treating atrial arrhythmias ${ }^{11,12}$ and heart failure ${ }^{13-15}$, while preclinical data suggests vagal stimulation has cardioprotective effects in models of pressure and ischemia by mitigating intrinsic and sensory neuron remodeling and cardiac hypertrophy ${ }^{16-18}$.

However, the fiber types and mechanisms that underlie the functional effects of VNS are either unknown or poorly understood. Given the range of organs which are innervated by the vagus nerve and the non-selectivity of electrode stimulation, many off-target fibers are stimulated for the therapies mentioned above. Adverse effects arising from VNS therapy include pain, paresthesia, hoarseness, voice alteration, cough, dyspnea, nausea and headache $e^{5,19,20}$.

\footnotetext{
${ }^{1}$ Departments of Bioengineering, University of Colorado - Anschutz Medical Campus, Aurora, CO, USA. 'Departments of Cell and Developmental Biology, University of Colorado - Anschutz Medical Campus, Aurora, CO, USA. ${ }^{3}$ Biomechatronics Development Laboratory, University of Colorado - Anschutz Medical Campus, Aurora, CO, USA. 'UCLA Cardiac Arrhythmia Center, University of California Los Angeles, Los Angeles, CA, USA. ${ }^{5}$ UCLA Neurocardiology Research Program of Excellence, University of California Los Angeles, Los Angeles, CA, USA. ${ }^{6}$ Rocky Mountain Veterans Affairs Medical Center (VAMC), Aurora, CO, USA. ${ }^{7}$ Division of Pharmacology, Department of Diagnostic and Therapeutic Sciences, Meikai University School of Dentistry, Saitama, Japan. ${ }^{8}$ These authors contributed equally: Arjun K. Fontaine and Gregory L. Futia. ${ }^{9}$ These authors jointly supervised this work: Diego Restrepo, John H. Caldwell, Emily A. Gibson and Richard F. ff Weir. ${ }^{\circledR}$ email: arjun.fontaine@cuanschutz.edu
} 
The limited mechanistic understanding and imprecise stimulation underlying current peripheral nerve therapies highlight the need for tools with greater selectivity and specificity. A potential avenue for achieving these improvements is optically mediated intervention using optogenetic tools ${ }^{21}$. With such techniques, light-driven activation or suppression of nerve pathways is possible with cell-type specific targeting ${ }^{22}$. In preclinical animal studies, virally-targeted excitatory opsins were employed in peripheral nerves to optically stimulate selective motor activity ${ }^{23,24}$, while inhibitory opsins have been used to suppress muscle activity ${ }^{23,25}$ and inhibit pain ${ }^{26}$. Adeno-associated viruses (AAVs) are widely used to deliver optical actuators and sensors in experimental models, with targeting specificity defined by tissue/location of delivery, virus serotype, and genetic promoter. There are numerous AAV-based gene therapies in human clinical trials, and to date, three have been approved for treatment in the U.S. and E. $\mathrm{U}^{27-30}$.

Given the advantages in precision that optical techniques offer, and the encouraging progress of AAV gene delivery in the clinical setting, we investigated the possibility of using retrograde AAV injection into an organ of interest to modulate its function with targeted optical stimulation in proximal nerve pathways. The current report presents a pilot study targeting the heart, which is regulated by extensive and complex control from the autonomic nervous system ${ }^{31,32}$. We demonstrate feasibility of this approach with injection of a retrograde AAV (rAAV2-retro) into the ventricular myocardium to transduce opsins proximally in fibers of the vagus nerve. Photostimulation of subsets of heart-specific axons was applied at the cervical vagus nerve using both one-photon illumination and two-photon holographic excitation ${ }^{33,34}$. Significant changes in heart rate and electrocardiogram (ECG) parameters, as well as the elicitation of respiratory reflex, serve as a proof-of-concept for organ-specific optogenetic neuromodulation and study of organ function.

\section{Methods}

Heart surgery and viral injection. The use of animals was approved by the Institutional Animal Care and Use Committee (IACUC) at the University of Colorado, Anschutz Medical Campus, with accreditation by the Association for Assessment and Accreditation of Laboratory Animal Care (AAALAC). All experiments were performed in accordance with IACUC regulations under an approved protocol. A total of 10 mice survived the relatively strenuous cardiac-injection surgical procedure and were tested in this pilot study. A high-level summary of results is shown in Supplementary Fig. 1. Three viral constructs were tested: $p A A V$-Syn-Chronos-GFP (59170$\mathrm{AAVrg})^{35}$, pAAV-Syn-ChR2(H134R)-GFP (58880-AAVrg) ${ }^{36}$, and AAV-CAG-hChR2-H134R-tdTomato (28,017AAVrg) ${ }^{37}$. These were packaged in AAV-retrograde (rAAV2-retro), a virus designed for efficient retrograde access to projection neurons ${ }^{38}$, and obtained from Addgene. The injected titers were approximately $\sim 1 \times 10^{12} \mathrm{vg} /$ $\mathrm{mL}$, and a total of $20 \mu \mathrm{l}$ volume was injected in each animal/procedure.

Mice (C57BL/6) were given carprofen $\left(5 \mathrm{mg} \mathrm{kg}^{-1}\right.$, s.c.) and buprenorphine $\left(0.05 \mathrm{mg} \mathrm{kg}^{-1}\right.$, s.c.) $1 \mathrm{~h}$ prior to surgery. Animals were anesthetized with isoflurane (induction at $5 \%$, maintenance at $1-3 \%$, inhalation), intubated, and mechanically ventilated (CWE Inc., SAR-830/AP). Core body temperature was measured and maintained at $37^{\circ} \mathrm{C}$. The surgical incision site was cleaned 3 times with $10 \%$ povidone iodine and $70 \%$ ethanol in $\mathrm{H}_{2} \mathrm{O}$ (vol/ vol). A left lateral thoracotomy was performed at the fourth intercostal space, the pericardium opened, and the heart was exposed. One microliter AAV stock $\left(\sim 1 \times 10^{13} \mathrm{vg} / \mathrm{mL}\right)$ diluted in $10 \mu \mathrm{L} 0.01 \mathrm{M}$ phosphate-buffered saline was subepicardially injected into each the left and right ventricle with a 31-gauge needle. The surgical wounds were closed with 6-0 sutures. Buprenorphine $\left(0.05 \mathrm{mg} \mathrm{kg}^{-1}\right.$, s.c.) was administered once daily for up to 2 days after surgery.

Vitals measurement. Mouse vitals (heart rate, pulse distension, breath rate, breath distension, pO2) were monitored and recorded in anesthetized mice with a MouseOx Plus suite (Starr Life Sciences) with a paw sensor. For ECG measurement, three needle electrodes were placed subdermally: on both sides of the upper chest cavity near the armpits and lead I in the lower abdomen. Signals were amplified (BioAmp) and digitized using a PowerLab 4/35 and were analyzed in LabChart software (ADInstruments).

One-photon photostimulation of cervical vagus nerve. Ten mice were tested in terminal procedures over a period of 6-23 weeks post viral injection, with robust expression and functional responses still observed at 23 weeks. Animals were anesthetized using 1-3\% isoflurane gas, maintained through nose cone inhalation, and placed supine on a heating pad, while vitals were monitored throughout the experiment. A $1 \mathrm{~cm}$ incision was made at the cervical region, 2-3 mm lateral to midline on the mouse's left side. Blunt dissection techniques were used to expose the left cervical vagus nerve and separate it from the carotid artery. An optical cannula (CFMC12L20, Thorlabs) was positioned with a micromanipulator to abut the nerve for laser photostimulation. A $473 \mathrm{~nm}$-wavelength solid-state laser (BL473T8-150FC, Shanghai Laser \& Optics Century) was coupled to the optical cannula with an optical patch cable $(200 \mu \mathrm{m}$ core, $0.39 \mathrm{NA}$, M81L005, Thorlabs) and interconnect (ADAF2, Thorlabs). The laser was controlled with a custom Arduino circuit board to output $5 \mathrm{~ms}$ pulses at $20 \mathrm{~Hz}$.

GRIN lens-integrated cervical nerve cuff. The nerve cuff was fabricated from silicone (Silastic MDX44210, Dow Corning) by compression molding. Mold components were designed in SolidWorks and fabricated using direct laser metal sintering (DLMS) in maraging steel. A GRIN lens, $9 \mathrm{~mm}$ in length, $1 \mathrm{~mm}$ in diameter (Inscopix 1050-002177), was placed in a polyimide tube (7.5 mm length, $1 \mathrm{~mm}$ inner diameter, $1.15 \mathrm{~mm}$ outer diameter, Microlumen Inc.) and fixed with cyanoacrylate. This assembly was inserted into the silicone cuff at a perpendicular orientation to the nerve's longitudinal axis, with the GRIN lens inset $250 \mu \mathrm{m}$ from the interior surface of the cuff, and fixed with cyanoacrylate. The cuff was fully assembled before implanting in the animal (Fig. 1A,B). 


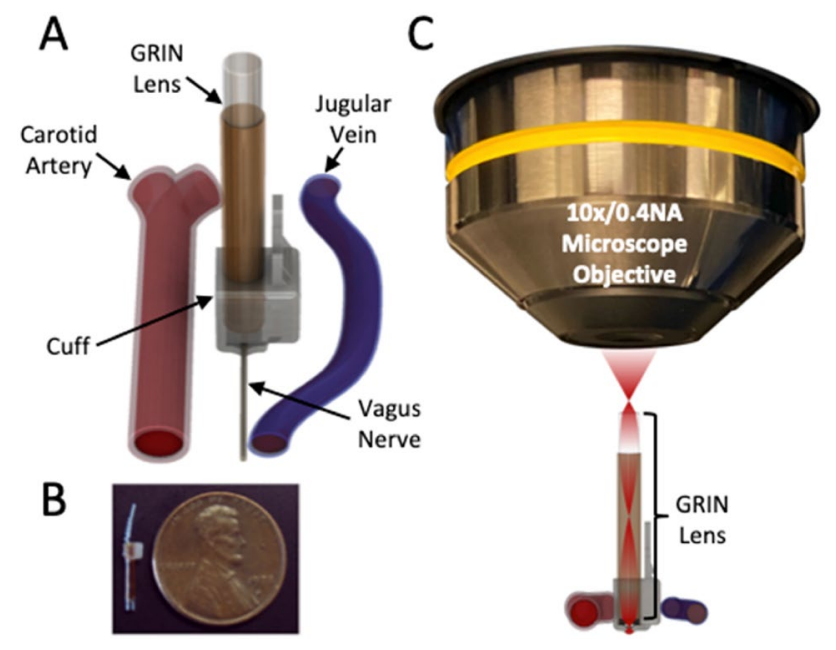

Figure 1. Detailed schematic of the GRIN-lens-integrated nerve cuff for two-photon imaging and holographic stimulation. (A) GRIN-cuff situated on the cervical vagus nerve between the carotid artery and jugular vein. (B) Photo of device next to penny for scale, in the un-cuffed (non-ratcheted) position. (C) Schematic of GRIN-cuff and microscope objective configuration.

Two-photon imaging and holographic photostimulation. The GRIN lens-integrated nerve cuff was fastened to the vagus nerve of the anesthetized mouse with a ratcheting strap and held rigid using custommodified dental forceps mounted to a three-axis manipulator. The mouse was placed supine with the GRIN lens positioned under the objective lens of an upright microscope for two photon imaging and holographic photostimulation (Fig. 1C).

A custom setup for two-photon imaging and holographic photostimulation was built on a Movable Objective Microscope (Sutter Instruments, Inc.), as described previously ${ }^{34}$ and shown schematically in Supplementary Fig. 2. A Ti:Sapphire laser oscillator (MaiTai HP, Newport, Inc.) producing $\sim 80 \mathrm{fs}$ pulses at $920 \mathrm{~nm}$ with an $80 \mathrm{MHz}$ repetition rate provided the excitation light for two-photon imaging. The GRIN lens (0.5 NA, 1:1 magnification) served as an optical relay, such that the cervical vagus nerve was in the conjugate plane of the focus of the objective lens (0.4 NA, Olympus USLSAPO10X2). Fluorescence emission from the sample was collected in the epi-direction, filtered by a dichroic (LP665) and bandpass filter (green channel, 510/42 and red channel, $620 / 60)$ and detected on large-area photomultipliers (10770PA-40, Hamamatsu). Typical imaging frame rates were $3.5-5.5 \mathrm{~Hz}$ with an imaging field of view of $\sim 200 \mu \mathrm{m}$.

Two-photon photostimulation was performed using a $1030 \mathrm{~nm}$ pulsed laser (SpectraPhysics, Spirit HE 103070) producing pulses with $\sim 240 \mathrm{fs}$ pulse duration at $500 \mathrm{kHz}$ repetition rate. The photostimulation beam path included a spatial light modulator (SLM, Phasor, 3i, Inc.) to create a desired spatial pattern at the objective focus ${ }^{39}$. The $1030 \mathrm{~nm}$ laser beam was expanded to fill the active area of the SLM and adjustable phase patterns were calculated in software (Slidebook 6, 3i, Inc.) and output onto the SLM. The $1030 \mathrm{~nm}$ laser was combined with the $920 \mathrm{~nm}$ laser using a polarization beam splitter placed directly after the scan lens. The SLM was imaged onto the back aperture of the objective lens at $1.6 \times$ magnification to slightly over fill the aperture. The phase pattern imparted on the beam by the SLM results in an intensity pattern at the focus of the objective. The spatial pattern at the sample was verified using a flat fluorescence slide detected on a widefield imaging system. An integrated acousto-optic modulator (AOM) in the $1030 \mathrm{~nm}$ laser was triggered with a function generator to control the timing for photostimulation. The setup is optimized for two-photon photostimulation of red-shifted opsins such as Chronos or ChrimsonR combined with imaging of reporter proteins such as GCaMP6. Additionally, the same setup was utilized for photostimulation of Channelrhodopsin2 (ChR2) at $920 \mathrm{~nm}$. Here, the signal output from a noncollinear optical parametric amplifier (NOPA-VIS-IR, Newport, Inc) tuned to $920 \mathrm{~nm}$ with a pulse duration of $\sim 50 \mathrm{fs}$ and repetition rate of $500 \mathrm{kHz}$, was sent through the same SLM beam path for holographic photostimulation.

GRIN lens aberrations. The GRIN relay lens introduces aberrations that can distort the laser focus offaxis. For two-photon imaging, this results in a fall off in intensity off axis. The typical field of view observed was approximately $200 \mu \mathrm{m}$ in diameter, encompassing the full mouse cervical vagus nerve. Aberrations also expand the axial confinement of the holographic stimulation and this becomes worse for photostimulation regions which are off axis ${ }^{40}$. We fixed the spacing from the imaging plane in the nerve to the GRIN lens surface at the optimal working distance of $300 \mu \mathrm{m}$ using the cuff. However, any deviation from the working distance can introduce further aberrations and reduce axial confinement ${ }^{41}$.

Spatial localization of 2-photon photostimulation. The two-photon excitation spatial profiles used for the animal experiments were characterized experimentally. A thin fluorescent sample on a slide was translated axially through the focus of the objective and GRIN relay lens and images of the fluorescent profile were 
acquired in a transmission microscope using a $10 \times 0.4 \mathrm{NA}$ objective and imaged on a CMOS camera. In this manner, the spatial profile in $\mathrm{x}, \mathrm{y}$, and $\mathrm{z}$ were fully mapped. Supplementary Fig. 3 shows the profiles used for the two-photon photostimulation reported in the Results section. Note that the axial extent of excitation increases with increasing lateral illumination area when performing holographic stimulation ${ }^{42}$. Better axial sectioning can be accomplished using temporal focusing that was not used in these experiments ${ }^{39}$.

Scattering of light in biological tissue also plays a role in the intensity pattern in the nerve and can limit the achievable depth. Additionally, distortions in the wavefront due to the heterogeneous tissue will also change the spatial excitation pattern in the nerve. In the future, implementing a method to characterize the intensity in vivo such as the technique by Lerman et al. ${ }^{43}$ will be important for precise control of illumination in this type of application.

Tissue histology. Animals were euthanized with urethane $(2 \mathrm{~g} / \mathrm{kg}$, i.p.). With a small-gauge needle inserted into the apex of the left ventricle, and incision made in the vena cava, animals were transcardially perfused with $50 \mathrm{ml}$ ice-cold phosphate-buffered saline (PBS) containing $100 \mathrm{U}$ heparin followed by $50 \mathrm{ml}$ of $4 \%$ paraformaldehyde (PFA) in PBS. Vagus nerves, nodose ganglia and superior cervical ganglia were excised and post-fixed in $4 \%$ PFA for $20 \mathrm{~min}$, then rinsed in PBS. Tissues were slide-mounted with standard mounting media and imaged for examination of reporter expression on a confocal spinning disk microscope (Marianas, Intelligent Imaging Innovations).

Brains were harvested and post-fixed overnight on ice before cryoprotection by incubation in $0.1 \mathrm{M}$ PBS with $30 \%$ sucrose overnight at $4{ }^{\circ} \mathrm{C}$. Brains were placed in a positional mold and cut transversally (coronal sections) in a Leica cryostat (Leica CM1900, Leica Biosystems, Nussloch gmbh, Germany) at $30 \mu \mathrm{m}$ thickness. All sections were imaged on a Nikon A1R microscope (Tokyo, Japan) with a $40 \times$ objective 1.3 N.A.

Brain sections were washed three times in PBS before antigen retrieval permeabilization (sodium citrate $10 \mathrm{mM}$, Tween $0.5 \%$, antigen retrieval $\mathrm{pH} 6.0$, Bio wave $550 \mathrm{~W}, 32^{\circ} \mathrm{C}, 5 \mathrm{~min}$ ). Sections were then permeabilized with $1 \%$ Triton X-100 in PBS for 30 min. Sections were washed three times in PBS between each permeabilization process.

After permeabilization, all slices were subjected to a blocking step for $1 \mathrm{~h}$ in $5 \%$ normal donkey serum (NDS) including $0.3 \%$ tween in PBS before primary antibody incubation overnight at room temperature. The following primary antibodies were used and diluted in 5\% NDS: goat anti-ChAT antibody (AB144P, 1:200) and chicken anti-GFP (Aves, GFP-1020, 1:500). Sections were washed three times in PBS before incubation in secondary antibodies diluted in 5\% NDS for $2 \mathrm{~h}$ at room temperature. The following secondary antibodies were used: donkey anti-goat Alexa Fluor 594 (1:500) and donkey anti-chicken Alexa Fluor 488 (1:500). Sections were counterstained with Nissl (NeuroTrace 640/660 Deep-Red Fluorescent Nissl Stain-Solution in DMSO, 1:400) for $1 \mathrm{~h}$ at room temperature, and washed three times in PBS before sealing with a cover glass.

For whole-mount heart staining, fixed hearts were blocked in $0.01 \mathrm{M}$ PBS with $10 \%$ NDS and $0.2 \%$ Triton $\mathrm{X}-100 \mathrm{PBS}$ for $6 \mathrm{~h}$ at room temperature with agitation. Tissues were then incubated in primary antibody diluted in $0.01 \mathrm{M}$ PBS with $0.2 \%$ Triton X-100 and $0.01 \%$ sodium azide for 3 nights at room temperature with agitation. The following primary antibody was used: chicken anti-GFP (Aves, GFP-1020, 1:1000). Tissues were washed several times in $0.01 \mathrm{M}$ PBS overnight before incubation in secondary antibodies diluted in $0.01 \mathrm{M}$ PBS with $0.2 \%$ Triton X-100 and $0.01 \%$ sodium azide for 2 nights at room temperature with agitation. The following secondary antibodies were used: donkey anti-rabbit Cy3 (Jackson ImmunoResearch, 711-165-152, 1:400), donkey anti-chicken 647 (Jackson ImmunoResearch, 703-605-155, 1:400), donkey anti-sheep Cy3 (Jackson ImmunoResearch, 713-165-003, 1:400), and donkey anti-goat Cy3 (Jackson ImmunoResearch, 705-165-003, 1:400). Whole hearts were washed several times in 0.01 M PBS overnight before being mounted on microscope slides in RIMS.

Statistics. For statistical group comparisons two-tailed t-tests were used. A two-sample unpaired t-test was used for independent groups, and a paired t-test was used for paired group data.

\section{Results}

Retrograde transduction of nerve and ganglia with heart-injected rAAV2-retro. Expression of the virally transduced opsin-fluorophore construct was observed in proximal nerve/ganglia targets for the three rAAV2-retro constructs injected: (1) Syn-Chronos-eGFP, (2) Syn-ChR2(h134R)-eGFP, and (3) CAGChR2(h134R)-tdTomato (Fig. 2). A subset of axons in the left and right cervical vagi had robust reporter expression, and labeled axons appeared to be non-fasciculated and distributed across the nerve. A sparse subset of neurons was also labeled within the nodose ganglia, indicating the uptake in afferent (sensory) fibers. Superior cervical ganglia (SCG) were examined in a subset of mice, and all contained sparse neuronal labeling (Fig. 2B). Viral injections of construct \#1 were delivered in transgenic ChAT-tdTomato mice which provided a cholinergic label, while the other constructs were injected in wild-type mice. In the ChAT-tdTomato-injected mice, viral expression was distributed across primarily non-cholinergic axons with less expression in ChAT-positive axons, suggesting an afferent-dominated expression profile (Fig. 2A). In these mice, the nerve contained an average of $25.2 \pm 2.3$ axons labeled with the viral reporter, of which $6.8 \pm 1.7(27 \%, \mathrm{n}=3)$ were co-labeled with the transgenic ChAT reporter.

Retrograde transduction in the brainstem with heart-injected rAAV2-retro. rAAV2-retro reporter expression was observed in axons and a small number of cells in the nucleus of the solitary tract (NTS) of the dorsal brainstem (Fig. 3). Cholinergic neurons were visualized by either expression of the transgenic ChAT-tdTomato reporter or by ChAT immunofluorescence with Alexa Fluor 594. Viral eGFP expression was found in axons, without cholinergic co-labeling, affirming the likelihood that these processes were afferent sen- 
A Syn-Chronos-eGFP in transgenic ChAT-tdTomato mouse

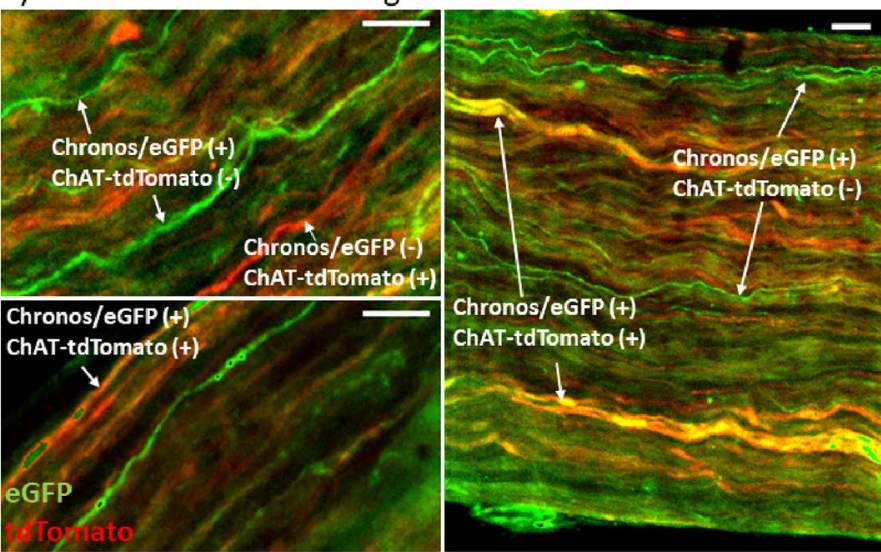

Vagus Nerve (Cervical)

B Syn-ChR2(h134R)-eGFP in WT mouse

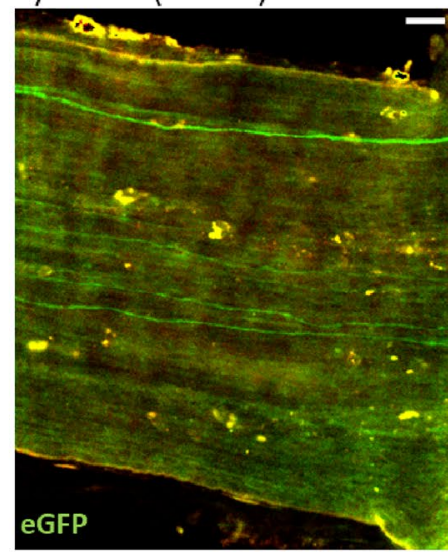

Vagus Nerve (Cervical)

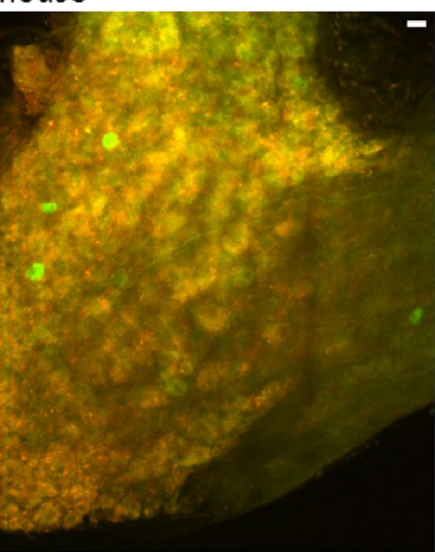

Nodose Ganglion

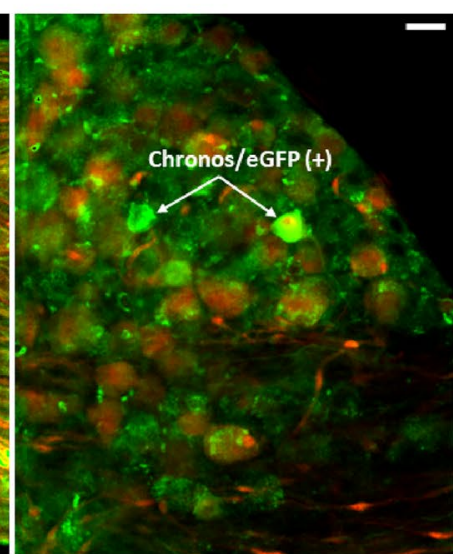

Nodose Ganglion

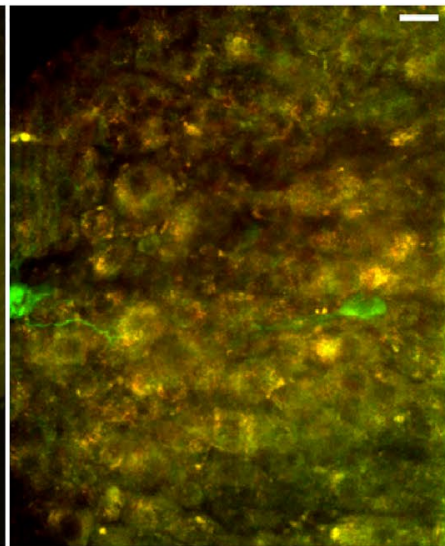

Superior Cervical Ganglion

C CAG-ChR2(h134R)-tdTomato in WT mouse

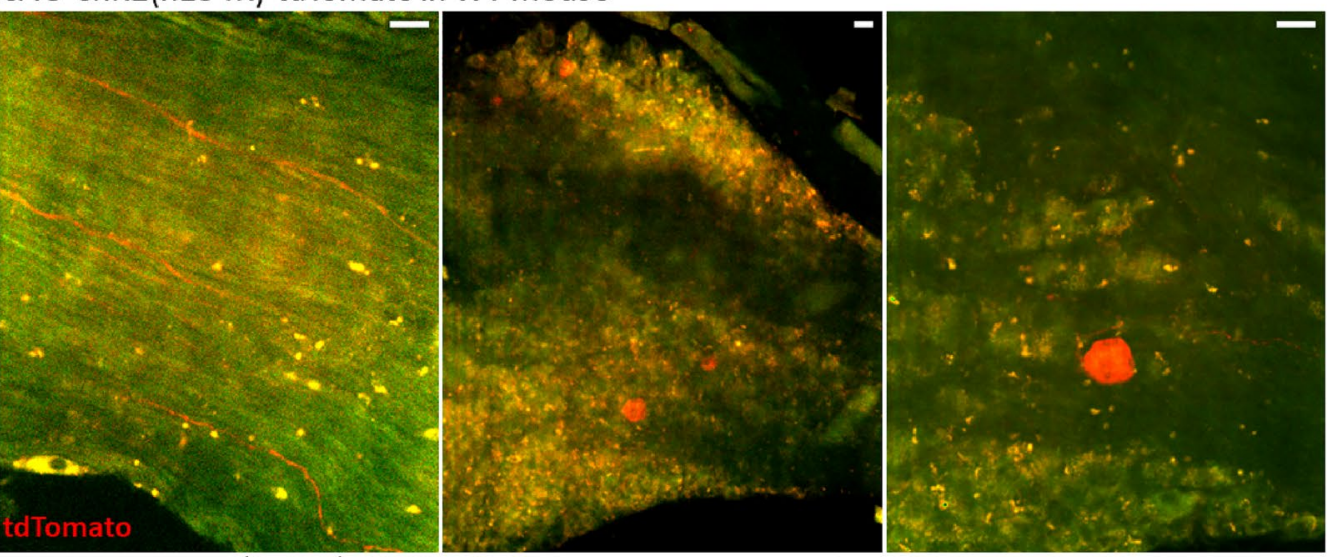

Vagus Nerve (Cervical)

Nodose Ganglion

Figure 2. Retrograde labeling in vagus nerve and nodose ganglia (NG) and superior cervical ganglia (SCG) with heart-injected rAAV2-retro constructs. Three constructs were injected: (A) Syn-Chronos-eGFP, injected in ChAT-tdTomato transgenic mice. eGFP expression (green) was observed in ChAT-positive (tdTomato, red) and ChAT-negative axons throughout the vagus nerve, and in neurons of the NG. (B) Syn-ChR2(h134R)-eGFP (green) injected in wild-type mice. eGFP expression is found in axons of the cervical vagus and NG neurons, as well as in neurons of the SCG. (C) CAG-ChR2(h134R)-tdTomato. tdTomato reporter expression (red) is observed in cervical vagus nerve and NG, in a similar expression pattern. All scale bars are $30 \mu \mathrm{m}$. (Images were rendered in Fiji software: https://imagej.net/Fiji). 
A
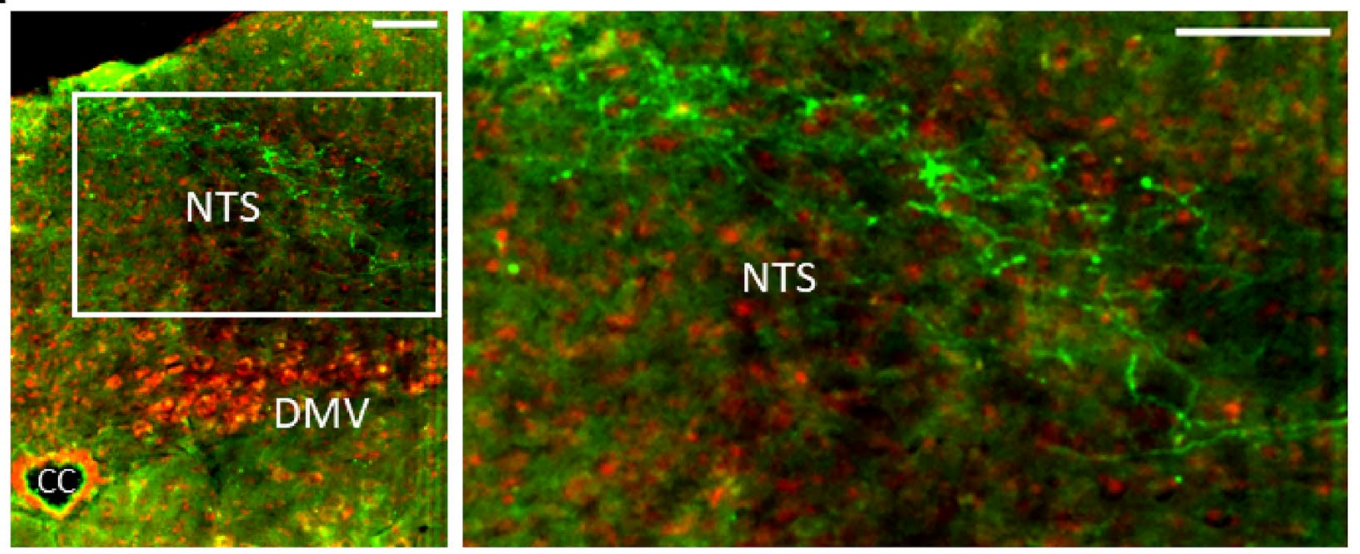

B

AAVrg-Syn-eGFP
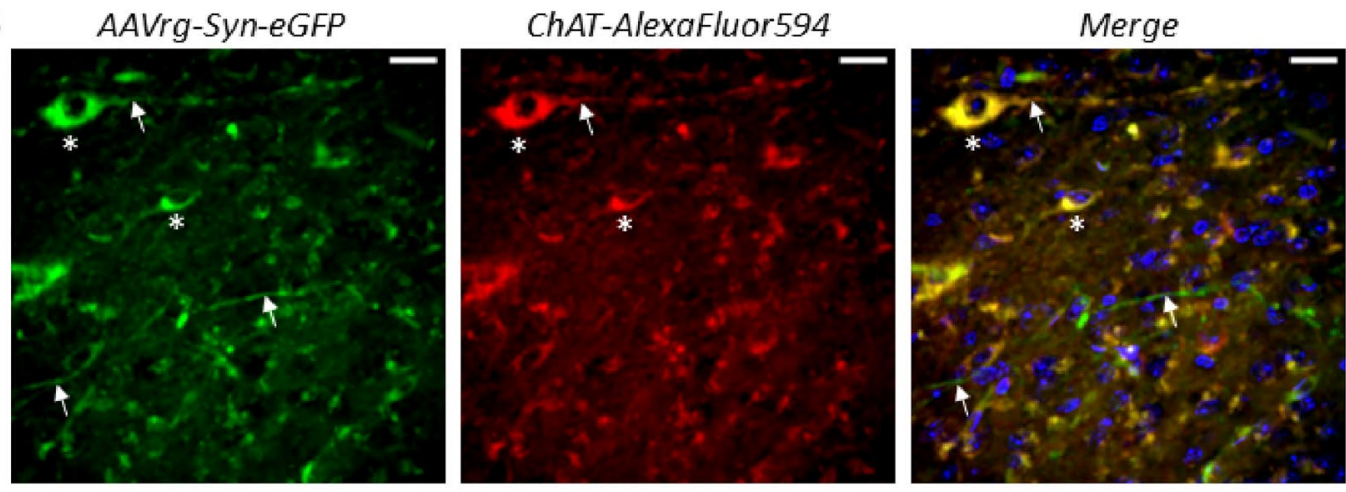

C

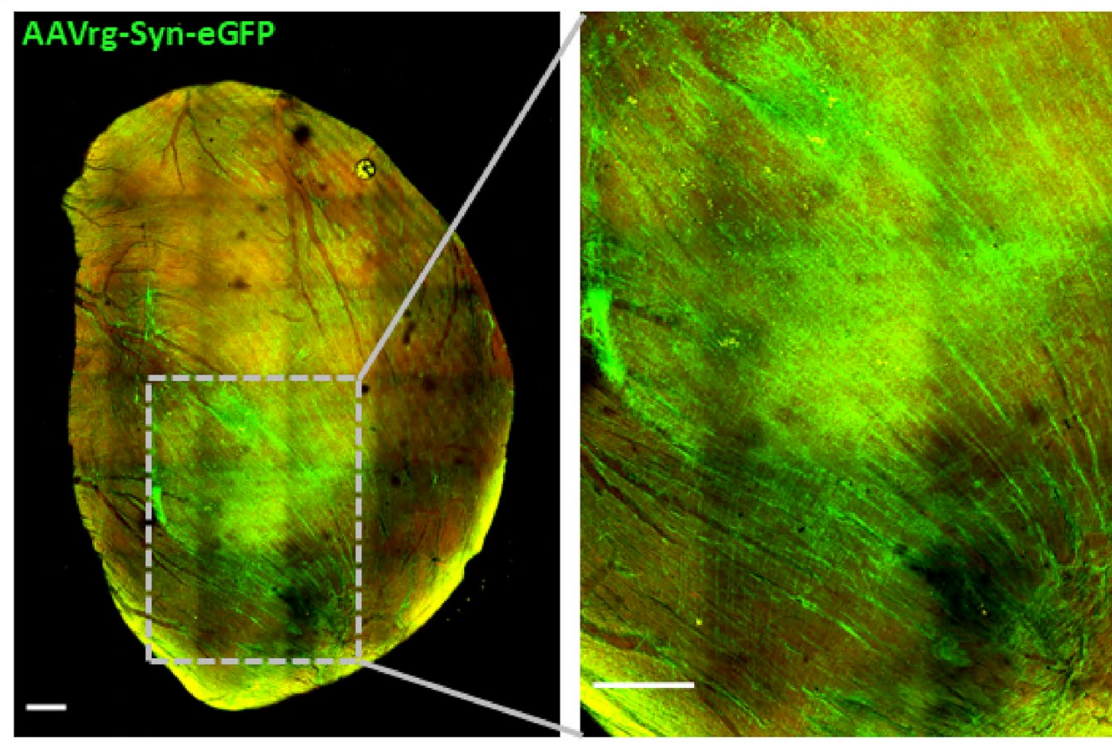

Figure 3. Retrograde viral reporter labeling in the dorsal brainstem and heart. (A) Representative confocal image showing eGFP in axonal processes (green) in the NTS in mice infected with AAVrg-Syn-ChR2(h134R)eGFP. Cholinergic neurons of the dorsal motor nucleus of the vagus (DMV) were immunolabeled with ChAT-AlexaFluor594 (red). CC: central canal (scale bar, $100 \mu \mathrm{m}$ ). (B) Virally transduced neurons expressing eGFP within NTS were also observed. Left: At least two neurons (white asterisks) as well as axons (arrows) are positively labeled with eGFP. Middle: ChAT antibody stains the eGFP-positive neurons. Right: Merged image along with Hoechst stain (blue) shows the co-labeled cholinergic neurons (yellow) and non-co-labeled axons (green) (scale bar, $20 \mu \mathrm{m}$ ). (C) eGFP reporter expression (green) in a heart injected with AAVrg-Syn-ChronoseGFP. Whole heart shown (left) with inset shown (right). (Images were rendered in Fiji software: https://image j.net/Fiji). 
A

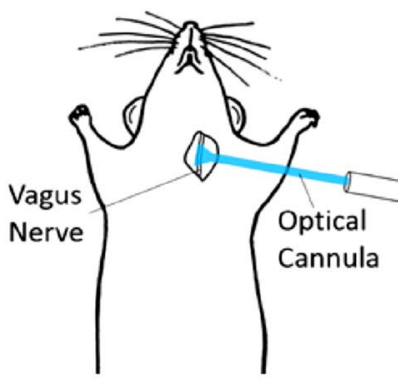

C

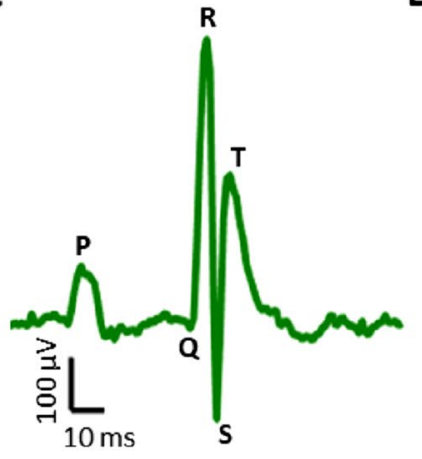

B Br Distension 15 $(\mu \mathrm{m})$

Hrt Rate

(bpm)
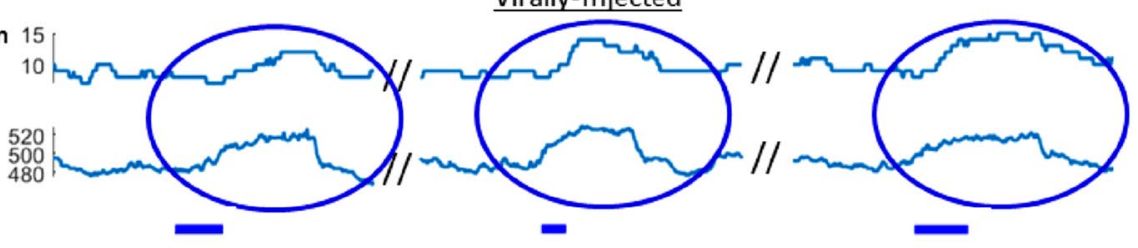

Non-Injected

Br Distension
$(\mu \mathrm{m})$
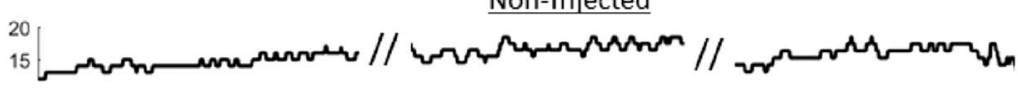

Hrt Rate

(bpm)
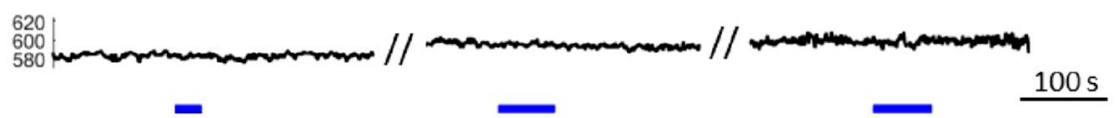

D

Virally-Injected

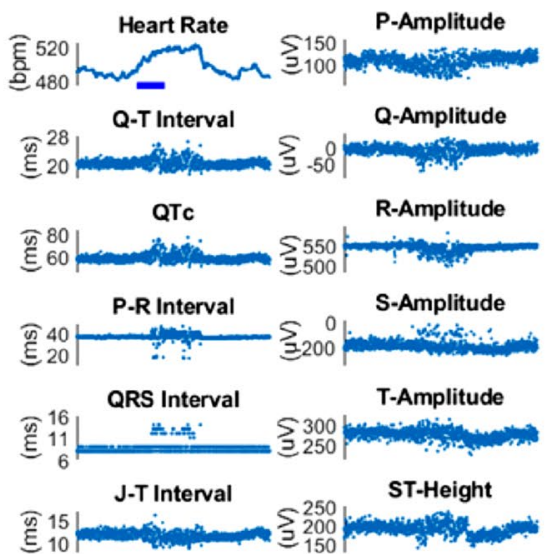

Non-Injected

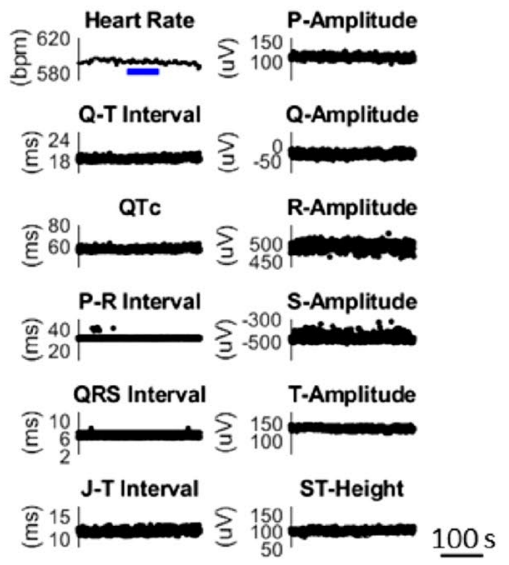

Figure 4. 1-Photon stimulation of the cervical vagus nerve perturbs vitals and ECG parameters. (A) Schematic of photostimulation in the anesthetized mouse. The left vagus nerve is exposed and illuminated with a $473 \mathrm{~nm}$ laser, delivered through an optical fiber/cannula. (B) Three consecutive photostimuli (blue bar) cause increases in heart rate and breath distension in a virally-injected (AAVrg-Syn-Chronos-eGFP) mouse while no changes occur in the non-injected control mouse. Note that the vertical axes in panels B and D show a limited range of values. (C) Representative ECG waveform (average of 4 beats). (D) Waveform parameters are modulated following stimulus onset through the duration of heart rate excursion in the virally-injected mouse while parameters are unmodulated in non-injected controls. (Blue bar: $473 \mathrm{~nm}, 5 \mathrm{~ms}$ pulses, $20 \mathrm{~Hz}, 10 \mathrm{~mW}$ unmodulated power).

sory projections (Fig. 3A). Interestingly, some sparse cholinergic neurons in NTS were also labeled with eGFP (Fig. 3B). Clear neuronal expression of viral reporters was not observed in the dorsal motor nucleus of the vagus (Fig. 3A) or the nucleus ambiguous (not shown). Reporter expression was also observed in the heart at the site of viral injection (Fig. 3C).

Photostimulation of the cervical vagus nerve in rAAV2-retro heart-injected mice. One-photon photostimulation of the left cervical vagus nerve in anesthetized mice was performed with a $473 \mathrm{~nm}$ laser via an optical fiber/cannula (Fig. 4A). Here, stimulation was non-selective and activated axons throughout the nerve cross-section. Mouse vitals (heart rate, breath rate, breath distension) as well as ECG parameters, were perturbed by optical stimulation. Response profiles were variable across mice however, with both increases and decreases in heart rate observed, with or without respiratory changes. This variability in functional responses could be explained by inconsistent viral uptake across fiber types due to injection-to-injection site variability. An example response profile is shown in Fig. 4; three consecutive stimuli ranging from 30 to $65 \mathrm{~s}$ in duration elicited reproducible, highly significant increases in heart rate and breath distension which arose during the optical stimulus and decayed to baseline 75-105 s following each stimulus (Fig. 4B). The onset of breath distension increase was delayed 20-30 s relative to the onset of heart rate change. The mean heart rate response had peak amplitude 17.6 standard deviations over baseline (pre-stimulation). Additionally, the peak amplitude is significantly greater than baseline (paired t-test, $p=5 \mathrm{e}-5, \mathrm{n}=3$ stimulations) and peak amplitude in non-injected controls (unpaired t-test, $p=4 \mathrm{e}-4, \mathrm{n}=3$ stimulations) (Supplementary Fig. 4B). The mean breath distension had peak amplitude 9.4 standard deviations over baseline (pre-stimulation), with peak amplitude significantly greater than baseline (paired t-test, $p=0.002, \mathrm{n}=3$ stimulations) and peak amplitude in non-injected controls (unpaired t-test $p=0.03$, $\mathrm{n}=3$ stimulations) (Supplementary Fig. 4B). Significant changes in ECG waveform parameters (representative ECG waveform shown in Fig. 4C) were observed concomitant with heart rate excursion (Fig. 4D). ECG param- 
eters had increased variance during the perturbed heart rate relative to pre and post-stimulus levels (see Supplementary Fig. 5). Changes in ECG variance in response to photostimulation were observed in mice throughout the study in which ECG was measured.

Two-photon holographic stimulation of cervical vagus nerve in rAAV2-retro heart-injected mice. Two-photon photostimulation of selected regions in the nerve was also performed. In addition to the heart-specific targeting achieved with retroviral delivery of the opsin, further selectivity is possible with spatial shaping of the excitation light. To this end, two-photon holographic photostimulation was employed. This approach enabled spatially differential photoexcitation as well as increased access within the nerve due to reduced tissue scattering of longer wavelength excitation light ${ }^{44}$. The implantation of a GRIN lens-incorporated cuff on the cervical vagus nerve enabled coupling of the nerve to a movable-objective-microscope (MOM) for in vivo two-photon imaging and photostimulation. A spatial light modulator (SLM) integrated into the microscope beam path provided the capability to illuminate spatially selected regions of interest within the nerve ${ }^{33}$.

Region-specific photostimulation in the nerve yielded robust changes in cardiac function as well as respiration. An example of perturbation in breath rate along with breath distension is shown in Fig. 5A, with null responses in non-injected controls shown in Fig. 5B. This mean breath rate reduction had peak amplitude 6.3 standard deviations over baseline (pre-stimulation) (paired t-test, $p=0.01, \mathrm{n}=3$ stimulations) and was significantly greater than non-injected controls (unpaired t-test, $p=0.02, \mathrm{n}=3$ stimulations), while mean breath distension had a peak signal 17.1 standard deviations over baseline (pre-stimulation) (paired t-test, $p=2 \mathrm{e}-4$, $\mathrm{n}=3$ stimulations) and was significantly greater than non-injected controls (unpaired t-test, $p=2 \mathrm{e}-4, \mathrm{n}=3$ stimulations) (Supplementary Fig. 4C). Differential responses in cardiac function were produced with differing spatial patterns of photostimulation. An example of this is presented in Fig. 5C,D, in which a broad stimulation region spanning the nerve cross section resulted in a depressive heart rate response that led to temporary heart block (Fig. 5C, peak signal reduction is 18.3 standard deviations over baseline), while more localized regions of stimulation caused an increase in heart rate along with an opposing set of ECG responses (Fig. 5D, peak signal increase is 7.1 standard deviations over baseline).

\section{Discussion}

This pilot study demonstrates improved targeting in peripheral nerve stimulation with organ-specific retrograde viral injection and optogenetic techniques. These results show the feasibility of targeted expression of opsins in vagal nerve fibers innervating the heart with a retrograde AAV virus injected into the myocardium. Cardiac viral injections elicited reporter expression in vagal axons, neurons within the nodose and superior cervical ganglia, and neuronal processes in the dorsal brainstem (Figs. 3, 4). One-photon photostimulation in the cervical vagus nerve elicited physiological responses (Fig. 4), and two-photon holography was employed to selectively stimulate subsets of vagal fibers, resulting in substantially different cardiorespiratory responses depending on which fibers were stimulated (Fig. 5). Unexpectedly, we also found expression of the reporter in cholinergic neurons in NTS (Fig. 3B). These findings demonstrate flexible alteration of cardiorespiratory function through targeted multiphoton cervical vagus holographic photostimulation. Additionally, the heart-specific opsin expression in vagal pathways achieved here with a retroviral approach will be applicable for neural targeting of other organ systems.

Vagal axons transduced by cardiac retrograde AAV delivery were determined to be primarily sensory afferents, since reporter expression was predominantly non-expressed in ChAT-driven fluorescently labeled fibers. It should be noted that labeling was likely limited in part by regionally specific injection of the AAV in the ventricles. Along with axonal labeling we found neuronal expression in the nodose ganglion and abundant axonal expression in NTS. Labeling of sparse cholinergic somata was also found in NTS. This raises the question of whether expression in somata occurred due to direct axonal innervation to the heart from NTS neurons, or by trans-synaptic hopping of the rAAV2-retro from a sensory afferent of the nodose ganglion to the NTS neuron. To our knowledge there is no report of trans-synaptic hopping by rAAV2-retro (personal communication, Alla Karpova and David Schaffer ${ }^{38}$ ). Thus, it is possible although unlikely, that viral reporter in ChAT-positive neurons in NTS may reflect labeling of NTS neurons innervating the heart. If these neurons also make local synaptic connections, this would be consistent with the finding by Furuya and colleagues that acetylcholine injected into the cNTS increases phrenic frequency and affects sympathetic-respiratory coupling, without changing sympathetic activity ${ }^{45}$. Alternatively, uptake of the virus into the systemic or pulmonary circulation could possibly account for this unexpected expression. Future experiments are necessary to investigate this.

While the work of this pilot study demonstrates vagal uptake and expression of opsins with cardiac injection, the targeting of fibers within the cardiorespiratory axonal subset was not highly specific. Future experiments should test methods for further honing specificity of expression. The precise location of viral delivery within the heart will likely have great influence on the specific populations of cardiorespiratory vagal axons that are transduced. For example, because most of the efferent vagal innervation is in the atria, viral injection here may yield a predominantly cholinergic (parasympathetic) uptake that may be therapeutically advantageous ${ }^{46,47}$. In addition to the location of injection, control of expression with a genetic promoter may ultimately provide the most specific targeting. The continued identification of molecular markers for vagal fibers with distinct physiological roles ${ }^{4-50}$ will provide an expanding library of axonal subsets that can be genetically targeted. Combining fibertype genetic control with organ and region specific viral delivery may lead to therapeutically precise treatment pathways with very little off-target activation.

Photostimulation of the cervical vagus nerve resulted in changes in heart rate. Interestingly, both increases and decreases in heart rate were observed. This could be explained by differing afferent populations expressing the opsin across experiments, as well as differential spatial stimulation in the case of the two-photon holographic stimulation. While reduced heart rate (bradycardia) is often associated with vagal stimulation, afferent-dominated 
A

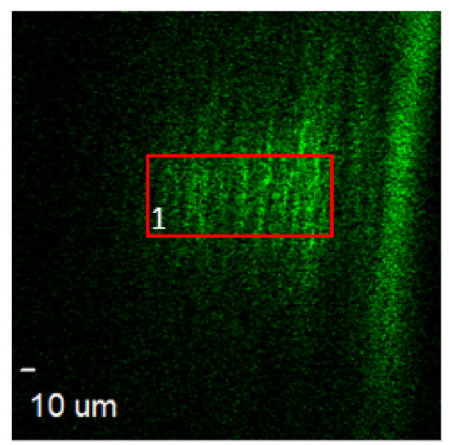

B

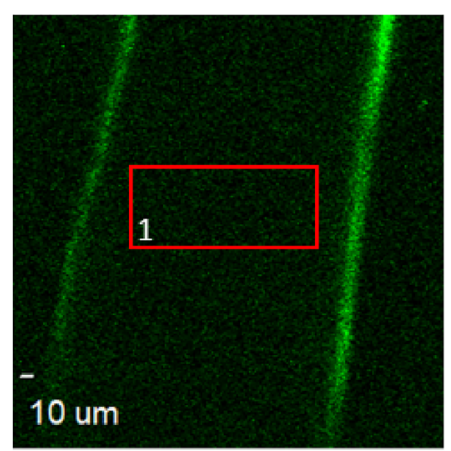

Virally-Injected
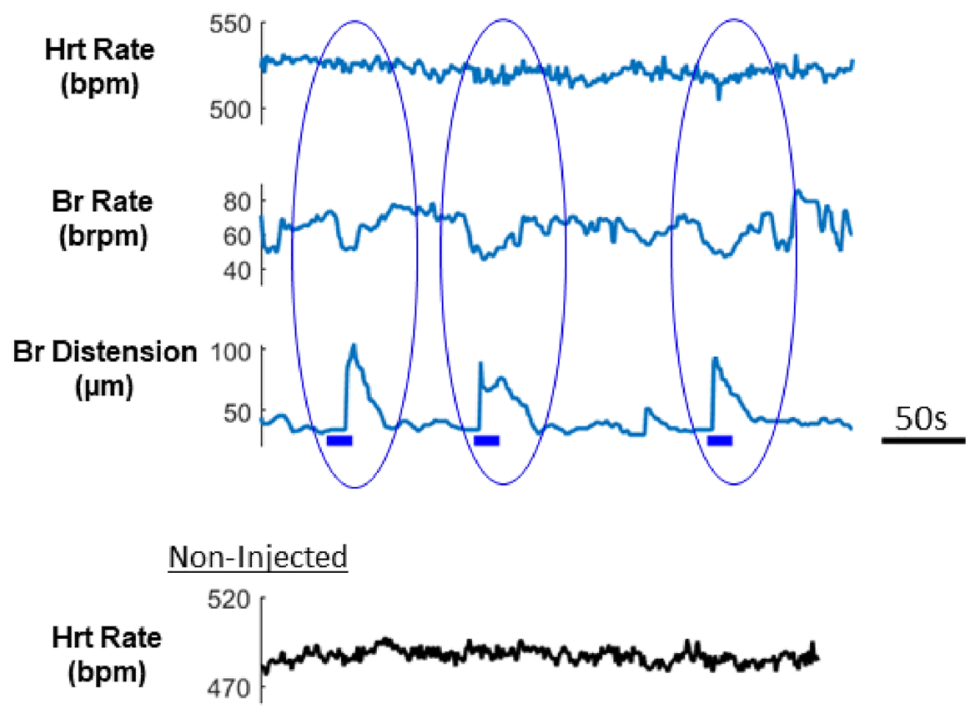

Br Rate

(brpm)

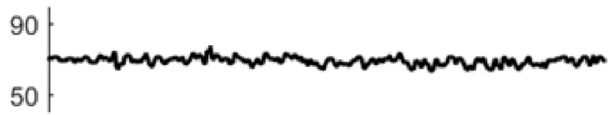

Br Distension

$(\mu \mathrm{m})$
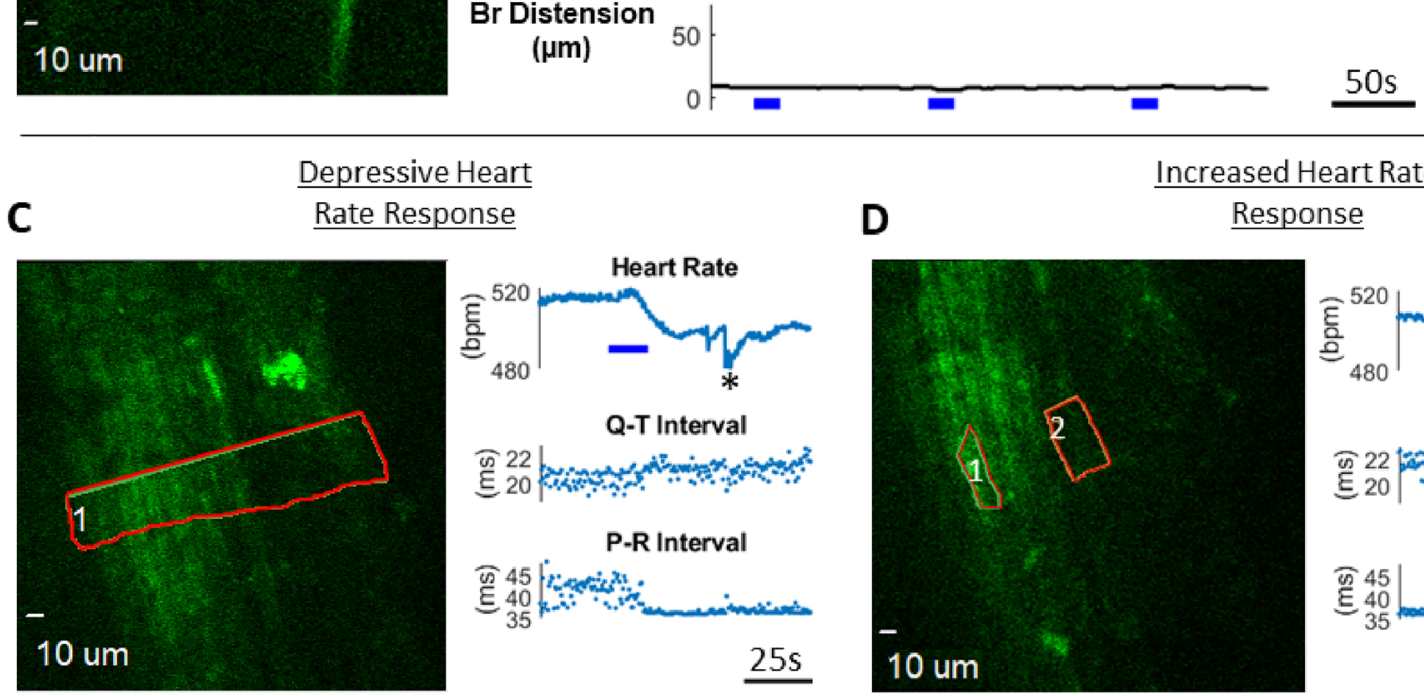

\section{onse}

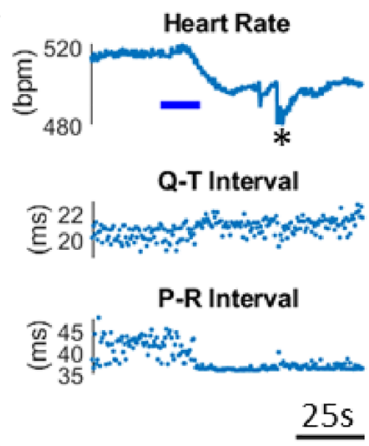

D

\section{$\underline{\text { Increased Heart Rate }}$}
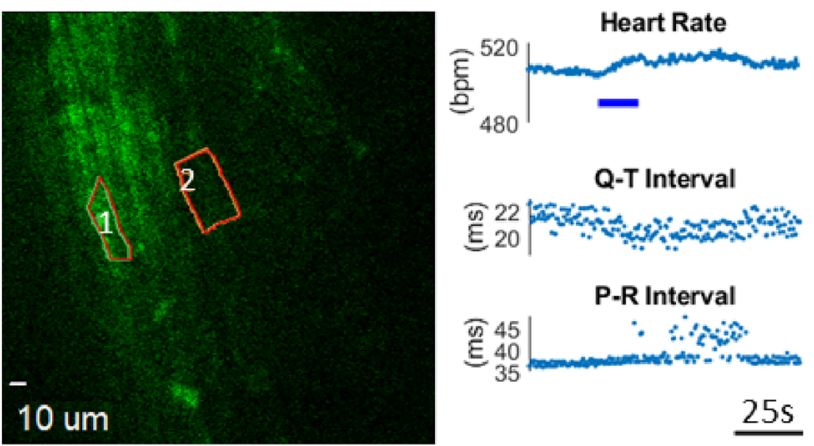

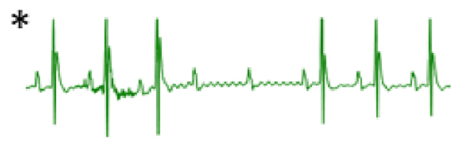

Figure 5. Two-photon holographic photostimulation in the cervical vagus nerve in mice infected with rAAV2retro in the heart and control. (A) $920 \mathrm{~nm}$ photoexcitation (red ROI) in AAVrg-CAG-ChR2(h134R)-tdTomatoinjected mouse elicited robust response in breath rate and distension (blue bar: $20 \mathrm{~Hz}$ pulses, $5 \mathrm{~ms}$ pulse duration, $0.014 \mathrm{~mW} / \mu^{2} \mathrm{~m}^{2}$ unmodulated intensity). (B) Same photoexcitation in non-injected control mouse did not evoke any respiratory or cardiac responses. (C, D) $1030 \mathrm{~nm}$ photoexcitation in AAVrg-Syn-Chronos-eGFPinjected mouse elicited differential heart response with spatially distinct photoexcitation regions (red ROIs). The broad photoexcitation region in panel $\mathrm{C}$ caused a depressive heart rate response that led to heart block (asterisk, see inset) while the excitation profile in panel D caused an increase in heart rate and opposing ECG parameter changes. (blue bar: $20 \mathrm{~Hz}$ pulses, $5 \mathrm{~ms}$ pulse duration, panel B: $0.12 \mathrm{~mW} / \mu \mathrm{m}^{2}$ unmodulated intensity and panel C: $0.18 \mathrm{~mW} / \mu \mathrm{m}^{2}$ unmodulated intensity). (Images were rendered in SlideBook6 software: https://www.intelligen t-imaging.com/slidebook). 
activation may lead to a suppression of efferent parasympathetic drive leading to increased heart rate ${ }^{51,52}$. Further optogenetic experiments may help identify which afferent species would account for tachycardiac responses. Differential afferent targeting with the vGlut1 and vGlut 2 mouse lines may be an appropriate starting point in sorting out these afferent populations as it has been shown that these afferents have distinct differences and may originate in different organ $s^{53}$. There are also reports of a small subset of sympathetic fibers that run with the vagus nerve $e^{54}$. The possibility of sympathetic fibers transduced by myocardial injection and causing tachycardia needs to be investigated further. ECG parameters were also perturbed during and following photostimulation. In addition to cardiac-specific indices, respiratory changes also occurred in response to stimulation, as measured with breath rate and distension. Brainstem cardiorespiratory modulatory circuits are well documented, and vagal afferents within NTS are known to drive respiratory centers, including the ventral respiratory column (VRC) within the medulla ${ }^{55,56}$.

Two-photon holographic photostimulation was employed to selectively stimulate vagal activity. Using a custom microscope setup and GRIN-lens integrated nerve cuff, this stimulation elicited the cardiac and respiratory effects described above, and also activated differential and opposing heart rate and ECG changes with distinct stimulation regions within the vagus nerve. This modality of photostimulation could potentially be used in the future as an experimental tool which adds optical depth access in tissue, and spatial selectivity for increased precision in studying the neural pathways affecting organ function.

\section{Conclusion}

Given that improved targeting is needed to precisely study and modulate organ function, we have investigated mechanisms within the optical (optogenetic) approach to neuromodulation. Delivery of opsins to organ-specific and genetically defined fibers is a critical aspect to this strategy. The present study provides an initial demonstration of retrograde opsin delivery in an organ-specific manner to facilitate peripheral nerve photomodulation. Results showed the feasibility of perturbing organ function with this technique. This work did not delve into the precise mechanisms and neuronal subsets giving rise to the cardiac and respiratory responses. Future studies may investigate defined subsets of peripheral autonomic fibers to determine and correlate neuronal molecular markers to distinct cardiac/organ functionalities. Identifying these neuronal markers of autonomic function and characterizing their effect on the organ will be central in the development of neuromodulatory strategies.

Received: 28 September 2020; Accepted: 18 January 2021

Published online: 11 February 2021

\section{References}

1. Horn, C. C., Ardell, J. L. \& Fisher, L. E. Electroceutical targeting of the autonomic nervous system. Physiology 34, 150-162 (2019).

2. Birmingham, K. et al. Bioelectronic medicines: A research roadmap. Nat. Rev. Drug Discov. 13, 399-400 (2014).

3. Waltz, E. A spark at the periphery. Nat. Biotechnol. 34, 904-908 (2016).

4. Gurbani, S. et al. Neuromodulation therapy with vagus nerve stimulation for intractable epilepsy: A 2-year efficacy analysis study in patients under 12 years of age. Epilepsy Res. Treat. 2016, 1-5 (2016).

5. Binnie, C. D. Vagus nerve stimulation for epilepsy. Clin. Neurol. 51, 990-992 (2000).

6. Nahas, Z. et al. Two-year outcome of vagus nerve stimulation (VNS) for treatment of major depressive episodes. J. Clin. Psychiatry 66, 1097-1104 (2005).

7. Bonaz, B. et al. Chronic vagus nerve stimulation in Crohn's disease: A 6-month follow-up pilot study. Neurogastroenterol. Motil. 28, 948-953 (2016).

8. Koopman, F. A. et al. Vagus nerve stimulation inhibits cytokine production and attenuates disease severity in rheumatoid arthritis. Proc. Natl. Acad. Sci. U. S. A. 113, 8284-8289 (2016).

9. De Leeuw, P. W. et al. Sustained reduction of blood pressure with baroreceptor activation therapy: Results of the 6-year open follow-up. Hypertension 69, 836-843 (2017).

10. Horbach, T. et al. Closed-loop gastric electrical stimulation versus laparoscopic adjustable gastric band for the treatment of obesity: A randomized 12-month multicenter study. Int. J. Obes. 40, 1891-1898 (2016).

11. Salavatian, S. et al. Vagal stimulation targets select populations of intrinsic cardiac neurons to control neurally induced atrial fibrillation. Am. J. Physiol. Heart Circ. Physiol. 311, H1311-H1320 (2016).

12. Stavrakis, S. et al. Low-level vagus nerve stimulation suppresses post-operative atrial fibrillation and inflammation. JACC Clin. Electrophysiol. 3, 929-938 (2017).

13. Premchand, R. K. et al. Extended follow-up of patients with heart failure receiving autonomic regulation therapy in the ANTHEMHF study. J. Card. Fail. 22, 639-642 (2016).

14. Zannad, F. et al. Chronic vagal stimulation for the treatment of low ejection fraction heart failure: Results of the neural cardiac therapy for heart failure (NECTAR-HF) randomized controlled trial. Eur. Heart J. 36, 425-433 (2015).

15. Gold, M. R. et al. Vagus nerve stimulation for the treatment of heart failure: The INOVATE-HF trial. J. Am. Coll. Cardiol. 68, 149-158 (2016).

16. Beaumont, E. et al. Vagus nerve stimulation mitigates intrinsic cardiac neuronal remodeling and cardiac hypertrophy induced by chronic pressure overload in guinea pig. Am. J. Physiol. Heart Circ. Physiol. 310, H1349-H1359 (2016).

17. Beaumont, E. et al. Vagus nerve stimulation mitigates intrinsic cardiac neuronal and adverse myocyte remodeling postmyocardial infarction. Am. J. Physiol. Heart Circ. Physiol. 309, H1198-H1206 (2015).

18. Salavatian, S. et al. Thoracic spinal cord and cervical vagosympathetic neuromodulation obtund nodose sensory transduction of myocardial ischemia. Auton. Neurosci. Basic Clin. 208, 57-65 (2017).

19. Panebianco, M., Rigby, A., Weston, J. \& Ag, M. Vagus nerve stimulation for partial seizures (Review) Summary of Findings for the Main Comparison. (2015). https://doi.org/10.1002/14651858.CD002896.pub2.www.cochranelibrary.com.

20. Ben-Menachem, E. Vagus nerve stimulation, side effects, and long-term safety. J. Clin. Neurophysiol. 18, 415-418 (2001).

21. Birkner, E., Berglund, K., Klein, M. E., Augustine, G. J. \& Hochgeschwender, U. Non-invasive activation of optogenetic actuators. 1-12 (2016). https://doi.org/10.1117/12.2044157.Non-invasive.

22. Montgomery, K. L., Iyer, S. M., Christensen, A. J., Deisseroth, K. \& Delp, S. L. Beyond the brain : Optogenetic control in the spinal cord and peripheral nervous system. Sci. Transl. med. 8, 337-rv5-337-rv5 (2016). 
23. Maimon, B. E., Sparks, K., Srinivasan, S., Zorzos, A. N. \& Herr, H. M. Spectrally distinct channelrhodopsins for two-colour optogenetic peripheral nerve stimulation. Nat. Biomed. Eng. 2, 485-496 (2018).

24. Towne, C., Montgomery, K. L., Iyer, S. M., Deisseroth, K. \& Delp, S. L. Optogenetic control of targeted peripheral axons in freely moving animals. PLOS ONE 8, e72691 (2013).

25. Liske, H. et al. Optical inhibition of motor nerve and muscle activity in vivo. Muscle Nerve https://doi.org/10.1002/mus.23696 (2013).

26. Iyer, S. M. et al. Optogenetic and chemogenetic strategies for sustained inhibition of pain. Nat. Publ. Gr. https://doi.org/10.1038/ srep30570 (2016).

27. Hudry, E. \& Vandenberghe, L. H. Therapeutic AAV gene transfer to the nervous system: A clinical reality. Neuron 101, 839-862 (2019).

28. Naso, M. F., Tomkowicz, B., Perry, W. L. \& Strohl, W. R. Adeno-associated virus (AAV) as a vector for gene therapy. BioDrugs 31, 317-334 (2017).

29. Rodrigues, G. A. et al. Pharmaceutical development of AAV-based gene therapy products for the eye. Pharm. Res. 36, 1-20 (2019).

30. Caccomo, S. FDA approves innovative gene therapy to treat pediatric patients with spinal muscular atrophy, a rare disease and leading genetic cause of infant mortality. (2019). https://www.fda.gov/news-events/press-announcements/fda-approves-innov ative-gene-therapy-treat-pediatric-patients-spinal-muscular-atrophy-rare-disease.

31. Ardell, J. L. et al. Translational neurocardiology: Preclinical models and cardioneural integrative aspects. J. Physiol. 594, 3877-3909 (2016).

32. Shivkumar, K. et al. Clinical neurocardiology defining the value of neuroscience-based cardiovascular therapeutics. J. Physiol. 594, 3911-3954 (2016).

33. Ronzitti, E. et al. Sub-millisecond optogenetic control of neuronal firing with two-photon holographic photoactivation of Chronos. J. Neurosci. 37, 1246-1317 (2017).

34. Futia, G. L., Fontaine, A., Littich, S., McCullough, C., Restrepo, D., Weir, R., Caldwell, J. \& Gibson, E. A. In vivo holographic photostimulation and two photon GCaMP6 imaging of vagus nerve axons using a GRIN lens integrated nerve cuff. in Proceedings of SPIE: Optogenetics and Optical Manipulation 201928 (2019). https://doi.org/10.1117/12.2521830.

35. Klapoetke, N. C. et al. Independent optical excitation of distinct neural populations. Nat. Methods 11, 338-346 (2014).

36. Boyden, E. S., Zhang, F., Bamberg, E., Nagel, G. \& Deisseroth, K. Millisecond-timescale, genetically targeted optical control of neural activity. Nat. Neurosci. 8, 1263-1268 (2005).

37. Mao, T. et al. Long-range neuronal circuits underlying the interaction between sensory and motor cortex. Neuron 72, 111-123 (2011).

38. Tervo, D. G. R. et al. A Designer AAV variant permits efficient retrograde access to projection neurons. Neuron 92, 372-382 (2016).

39. Hernandez, O. et al. Three-dimensional spatiotemporal focusing of holographic patterns. Nat. Commun. 7, 1-11 (2016).

40. Accanto, N. et al. Multiplexed temporally focused light shaping through a gradient index lens for precise in-depth optogenetic photostimulation. Sci. Rep. 9, 7603 (2019).

41. Wang, C. \& Ji, N. Characterization and improvement of three-dimensional imaging performance of GRIN-lens-based two-photon fluorescence endomicroscopes with adaptive optics. Opt. Express 21, 27142-27154 (2013).

42. Lutz, C. et al. Holographic photolysis of caged neurotransmitters. Nat. Methods 5, 821-827 (2008).

43. Lerman, G. M., Little, J. P., Gill, J. V., Rinberg, D. \& Shoham, S. Real-time in situ holographic optogenetics confocally unraveled sculpting microscopy. Laser Photon. Rev. 13, 1900144 (2019).

44. Kobat, D. et al. Deep tissue multiphoton microscopy using longer wavelength excitation. Opt. Express 17, 13354-13364 (2009).

45. Furuya, W. I. et al. Differential modulation of sympathetic and respiratory activities by cholinergic mechanisms in the nucleus of the solitary tract in rats. Exp. Physiol. 99, 743-758 (2014).

46. Machhada, A. et al. Origins of the vagal drive controlling left ventricular contractility. J. Physiol. 594, 4017-4030 (2016).

47. Coote, J. H. Myths and realities of the cardiac vagus. J. Physiol. 591, 4073-4085 (2013).

48. Kupari, J., Häring, M., Agirre, E., Castelo-Branco, G. \& Ernfors, P. An atlas of vagal sensory neurons and their molecular specialization. Cell Rep. 27, 2508-2523.e4 (2019).

49. Chang, R. B., Strochlic, D. E., Williams, E. K., Umans, B. D. \& Liberles, S. D. Vagal sensory neuron subtypes that differentially control breathing. Cell 161, 622-633 (2015).

50. Williams, E. K. K. et al. Sensory neurons that detect stretch and nutrients in the digestive system. Cell 166, 209-221 (2016).

51. Ardell, J. L., Rajendran, P. S., Nier, H. A., KenKnight, B. H. \& Andrew Armour, J. Central-peripheral neural network interactions evoked by vagus nerve stimulation: Functional consequences on control of cardiac function. Am. J. Physiol. Heart Circ. Physiol. 309, H1740-H1752 (2015).

52. Ardell, J. L. et al. Defining the neural fulcrum for chronic vagus nerve stimulation: Implications for integrated cardiac control. J. Physiol. 595, 6887-6903 (2017).

53. Hermes, S. M., Colbert, J. F. \& Aicher, S. A. Differential content of vesicular glutamate transporters in subsets of vagal afferents projecting to the nucleus tractus solitarii in the rat. J. Comp. Neurol. 522, 642-653 (2014).

54. Seki, A. et al. Sympathetic nerve fibers in human cervical and thoracic vagus nerves. Hear. Rhythm 11, 1411-1417 (2014).

55. Alheid, G. F., Jiao, W. \& McCrimmon, D. R. Caudal nuclei of the rat nucleus of the solitary tract differentially innervate respiratory compartments within the ventrolateral medulla. Neuroscience 190, 207-227 (2011).

56. Zoccal, D. B., Furuya, W. I., Bassi, M., Colombari, D. S. A. \& Colombari, E. The nucleus of the solitary tract and the coordination of respiratory and sympathetic activities. Front. Physiol. 5, 1-12 (2014).

\section{Acknowledgements}

We thank the following funding institutions that supported this work: National Institutes of Health, Stimulating Peripheral Activity to Relieve Conditions (SPARC) initiative NIH OT2OD023852 (RFW, JHC, EAG), NIH U01NS099577 (DR, EAG), NIH NINDS R01NS118188-01 (Caldwell, Gibson, Weir): Optimization of a Minimally-Invasive Bidirectional Optogenetic Peripheral Nerve Interface with Single Axon Read-in \& Read-out Specificity, National Science Foundation NSF CBET-1631912 (EAG, DR), and Rocky Mountain Regional VA Medical Center R\&D Research Career Scientist Award IK6RX002996 (RFW). We thank Arianna Gentile Polese for immunohistochemistry help and Dominik Stich who performed imaging of whole hearts. We acknowledge the use of instruments in the Advanced Light Microscopy Core; part of the NeuroTechnology Center at University of Colorado Anschutz Medical Campus supported in part by Rocky Mountain Neurological Disorders Core Grant No. P30 NS048154 and by Diabetes Research Center Grant No. P30 DK116073.

\section{Author contributions}

A.K.F. performed the vagal stimulation experiments, tissue processing and imaging, analyzed the experimental data and drafted the manuscript. G.L.F. built and characterized the two-photon microscope and holography systems and operated these systems during experiments. P.S.R. performed the cardiac viral-injection surgeries 
and provided input on the manuscript. S.F.L. fabricated the GRIN-cuff device and participated in experiments. N.M. processed the brain tissue and performed immunohistochemical staining of tissues. K.S. and J.L.A. provided support and cardiac expertise in the investigation. D.R. and J.H.C. advised on viral constructs and transgenic animals. E.A.G. advised on the two-photon microscopy, holography and GRIN lens design and characterization. R.F.W. oversaw the development and fabrication of the nerve cuff device. R.F.W., E.A.G., J.H.C., and D.R. initiated and directed the study and edited the manuscript.

\section{Competing interests}

The authors declare no competing interests.

\section{Additional information}

Supplementary Information The online version contains supplementary material availlable at https://doi. org/10.1038/s41598-021-83280-3.

Correspondence and requests for materials should be addressed to A.K.F.

Reprints and permissions information is available at www.nature.com/reprints.

Publisher's note Springer Nature remains neutral with regard to jurisdictional claims in published maps and institutional affiliations.

Open Access This article is licensed under a Creative Commons Attribution 4.0 International License, which permits use, sharing, adaptation, distribution and reproduction in any medium or format, as long as you give appropriate credit to the original author(s) and the source, provide a link to the Creative Commons licence, and indicate if changes were made. The images or other third party material in this article are included in the article's Creative Commons licence, unless indicated otherwise in a credit line to the material. If material is not included in the article's Creative Commons licence and your intended use is not permitted by statutory regulation or exceeds the permitted use, you will need to obtain permission directly from the copyright holder. To view a copy of this licence, visit http://creativecommons.org/licenses/by/4.0/.

(C) The Author(s) 2021 